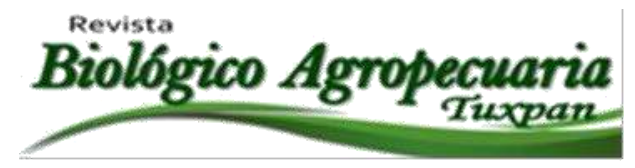

\title{
Venado cola blanca (Odocoileus virginianus) y cérvidos exóticos, en un sitio de la sierra nevada, Puebla, México
}

White-tailed deer (Odocoileus virginianus) and exotic deer, in a site of the sierra nevada, Puebla, Mexico

\author{
Salvador Romero-Castañón ${ }^{1 凶}$, Oscar Villarreal Espino Barros ${ }^{1}$; Roberto Resendíz \\ Martínez ${ }^{1}$ \\ ${ }^{1}$ Facultad de Medicina Veterinaria y Zootecnia. Benemérita Universidad Autónoma de Puebla. \\ Km. 7.5 Carretera Cañada Morelos-El Salado. C.P. 75470. Tecamachalco, Puebla. México. \\ Autor para correspondencia: salvador.romero@ correo.buap.mx
}

Recibido: $18 / 02 / 2017$

Aceptado: 30/06/2017

\section{RESUMEN}

La introducción de especies exóticas se considera como un factor importante que puede ocasionar la extinción, o declive de poblaciones. En el presente trabajo se realiza una estimación de la densidad poblacional del venado cola blanca (Odocoileus virginianus) nativo, y de cuatro especies de cérvidos exóticos que se introdujeron con fines cinegéticos en una Unidad de Manejo para la Conservación de la Vida Silvestre (UMA), ubicada en la Sierra Nevada de Puebla, México. Se utilizaron dos técnicas, una indirecta a través de excretas por el método de Eberhardt y Van Etten; y otra directa a través de avistamientos de individuos, utilizando el programa de computo Distance. Los resultados del primer método indican una densidad poblacional absoluta de 99 individuos; y en el segundo de 169 individuos. Por razones estadísticas; así como de un efectivo muestreo de excretas; se eligió como técnica más confiable el método indirecto; de tal manera de no sobre estimar la densidad poblacional de los cérvidos. Se discute de los daños ecológicos que pudieran generar la presencia de esos ungulados exóticos, y se sugiere erradicarlos del sitio.

Palabras clave: poblaciones, cérvidos, exóticos, UMA, daños ecológicos.

\begin{abstract}
The introduction of exotic species is considered as an important factor that provokes the extinction or decline of population. An estimation of the population density of white-tailed deer, that is native in the area, is being carried out in the present work, also are included four species of exotic deer that were introduced for hunting in a Unit Management and Sustainable Use of Wildlife, it is located in the Sierra Nevada of Puebla, Mexico. Two techniques were used, an indirect by means of feces by the Eberhardt and Van Etten method, and other direct through sightings of individuals, making use of computer program Distance. The results of first method indicate a total density of Ninety-nine individuals and the second one show a hundred and sixty-nine individuals. The result by indirect method
\end{abstract}


was chosen as the most reliable technique due to statistical issues; as well as effective sampling excreta, besides the population of deer is not overestimate. The ecological damages that could be caused by the presence of exotic deer are discussed and it is suggested to remove them from the area.

Keywords: populations, cervids, exotics, UMA, ecological damages.

\section{INTRODUCCÓN}

El creciente ritmo de extinción de especies, es resultado del incremento poblacional y las actividades humanas que amenazan a la biodiversidad por múltiples factores, que pueden ser directos o indirectos. Un factor que es tanto directo como indirecto es la introducción de especies exóticas; identificado como un aspecto importante relacionado con la extinción de especies. Algunos autores señalan, que la introducción de especies exóticas ocupa el segundo lugar de importancia en este rubro, después de la destrucción del hábitat, al actuar de manera sinérgica con otras amenazas (Alvarez et al., 2008).

La Unidad de Manejo para la Conservación de la Vida Silvestre (UMA), "San Gabriel Ixtapalucan", Puebla, México; en el año de 1996 con fines cinegéticos, se introdujeron cuatro especies de ciervos exóticos: venado gamo (Dama dama) cuya distribución original es Europa y Asia; venado axis (Axis axis), originario de Nepal, India y Sri Lanka; ciervo rojo (Cervus elaphus), originario de Europa y Asia; y el venado sika (Cervus nippon), originario de Rusia, Vietnam, Taiwán, China y Japón (Clutton-Brock, 2002., Alvarez et al., 2008). En ese sitio está presente un cérvido oriundo, el venado cola blanca (Odocoileus virginianus), subespecie mexicanus, conocido regionalmente como el "venado de los volcanes" (VIllarreal,
1998). El objetivo inicial en esta UMA fue el aprovechamiento cinegético, el cual hasta la fecha ha sido insuficiente y con poco éxito económico; además de no tener ningún otro tipo de aprovechamiento extractivo.

Las cinco especies de cérvidos se han mantenido en el predio, existiendo una relación interespecífica entre ellas (Fig.1). Se considera que los cérvidos exóticos pudieran estar ocasionando daños ambientales, además de generar un impacto negativo en la estructura y ecología de la población del venado cola blanca (Putman, 1988). Otros efectos negativos que se puede ocasionar la presencia de los exóticos son: posible introducción de enfermedades, y competencia por los recursos alimenticios con las especies nativas, que conlleva al desplazamiento de las mismas (Weber, 1993; VIllarreal, 1998); sobrepastoreo y posibles fugas de ejemplares que se convertirían en fauna perjudicial en el Parque Nacional Izta-Popo, ya que la UMA colinda con esta Área Natural Protegida (ANP) de la Sierra Nevada (Villarreal, 1998; Weber et al., 2006). Además, de afectar el hábitat por sobrepasar su capacidad de carga $(K)$ (Douglas et al., 2003).

Así mismo; contaminación genética a nivel de subespecies (Weber, 1993), ocasionando partos distócicos, prolapso vaginal y uterino, ruptura del útero $\mathrm{y}$ 
muerte de la madre y/o el producto; y alteración genética de la subespecie nativa por hibridación (Weber y GalindoLeal, 1992; Galindo-Leal y Weber, 1994; Villarreal, 1998).

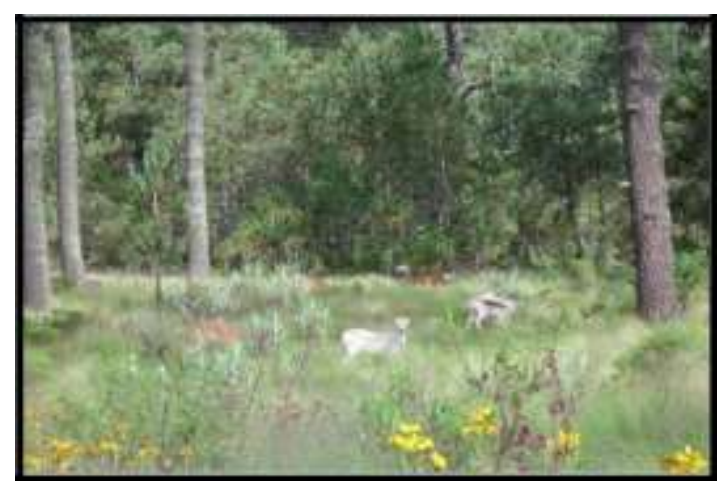

Figura 1. Presencia de cérvidos exóticos en el parque Izta-Popo

Existen dos tipos de estrategias ecológicas que utilizan las especies: la estrategia " $r$ ", que maximiza la capacidad reproductiva de los individuos y la tasa de crecimiento de la población, pero tiene una habilidad competitiva baja. La otra es la estrategia " $k$ " que maximiza la habilidad competitiva y la estabilidad de las poblaciones (Weber, 1993). Los ciervos de los géneros Odocoileus tienden a presentar estrategias " $r$ ", a diferencia de los géneros Cervus, Dama y Axis que poseen estrategias " $k$ " (Harrington, 1985); lo que implica una ventaja para los cérvidos exóticos, en estrategias reproductivas, selección del hábitat y habilidad para dispersarse. El venado cola blanca se cataloga como especie " $r$ " en relación con los ciervos del viejo mundo, debido a que este cérvido es un

ramoneador con alto potencial reproductivo; mientras que los exóticos tienen una adaptación considerable y tolerancia para utilizar alimentos fibrosos, como las gramíneas que poseen una relativa baja calidad nutricional (Weber, 1993; Van Soest, 1994; Ramírez, 2004).
Además, en la región de la Sierra Nevada hay un lagomorfo endémico, el conejo de los volcanes (Romerolagus diazi),

también llamado "teporingo" o "zacatuche", el cual se encuentra en peligro de extinción. Esta especie habita principalmente en los zacatones (gramíneas silvestres), que utilizan para hacer madrigueras, y se alimenta de brotes de herbáceas y gramíneas (Villarreal, 1998). La presencia de cérvidos exóticos representa también una competencia para el zacatuche ya que suelen ser de hábito pastoril, además de que generan compactación del terreno por el constante pisoteo de la zona, alterando así el hábitat de ese conejo, originando competencia por alimento, cobertura y espacio (Villarreal, 1998). Debido a la problemática antes descrita, el objetivo del presente artículo fue estimar la densidad poblacional del venado cola blanca y los cérvidos exóticos en esa UMA.

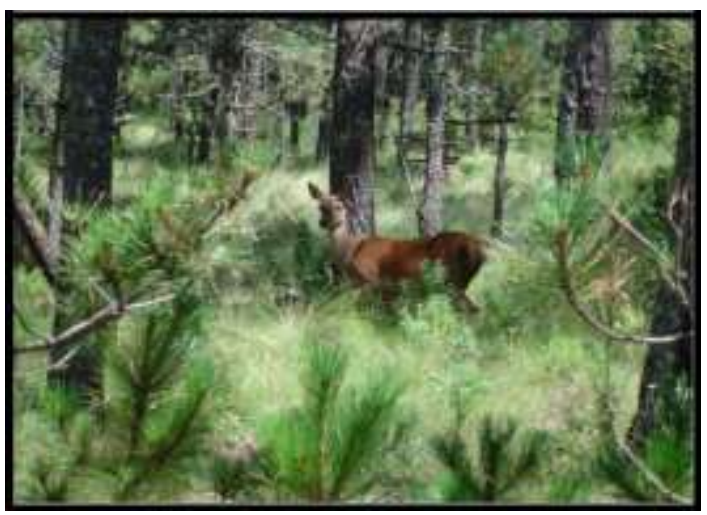

Figura 2. Hembra de Ciervo rojo (Cervus elaphus)

\section{Materiales y Método}

La UMA San Gabriel Ixtapalucan, se encuentra en la vertiente nororiental del volcán Iztaccíhuatl (5320 msnm), Puebla, México; el cual forma parte de la Sierra Nevada. La UMA se encuentra a una 
altitud promedio de $3041 \mathrm{msnm}$, con una extensión de 800 ha., en las coordenadas UTM: 0546271, 2122739, cuenta con vegetación de pino-encino y su clima es templado subhúmedo con lluvias en verano (INEGI, 2000). La UMA está cercada con malla ciclónica de dos metros de altura y colinda con la ANP, Parque Nacional Izta-Popo.

Se estimaron las densidades poblacionales del venado cola blanca y los cérvidos exóticos: venado gamo, venado axis, ciervo rojo y venado sika,; utilizando un método indirecto y otro directo. Como método indirecto se utilizó el modelo de Eberhardt y Van Etten (1956), mediante el conteo de grupos fecales en transectos. Como método directo se utilizó el transecto en línea, a través del programa de cómputo Distance $^{\mathrm{R}}$ (Thomas et al., 2005).

El método indirecto fue mediante el conteo de grupos fecales en transectos lineales. La fórmula para estimar la densidad es:

\section{(Dgf X Np) \\ $\mathrm{D}$ ind $=$ \\ (Tdf X Tdep)}

Donde D ind= la densidad de individuos por kilómetro cuadrado; Dgf = es la densidad promedio de grupos fecales por parcela de muestreo; $\mathrm{Np}=$ número de parcelas de muestreo que hay o caben en un kilómetro cuadrado; Tdf = tasa de defecación; y Tdep = días de depósito de los excrementos.

Los supuestos en que se basa esta metodología para estimar la densidad, son (Eberhardt y Van Etten 1956):
Conocer la tasa promedio de defecación de la especie de cérvido;

Conocer el tiempo de depósito de los grupos fecales.

Correcta identificación de esas excretas.

Eficiente tamaño de y forma de las

parcelas, para obtener un conteo preciso.

En el predio se trazaron cinco transectos al azar con una longitud de 800 metros cada uno. En cada transecto, se establecieron 40 parcelas de muestreo con una superficie de $10 \mathrm{~m}^{2}$ cada una, siendo un total de 200 parcelas. Las parcelas se revisaron a los 30 y 60 días después de que previamente habían sido limpiadas (barridas). La identificación de excretas de las diferentes especies de cérvidos fue a través de características físicas, como tamaño y morfología (Sanders, 1963); las cuales fueron determinadas de acuerdo a un patrón (Figura 3), a través de excretas colectadas con animales cautivos de criaderos y zoológicos; además de contar con el apoyo de los guías locales, que tienen una amplia experiencia en la identificación de las fecas.

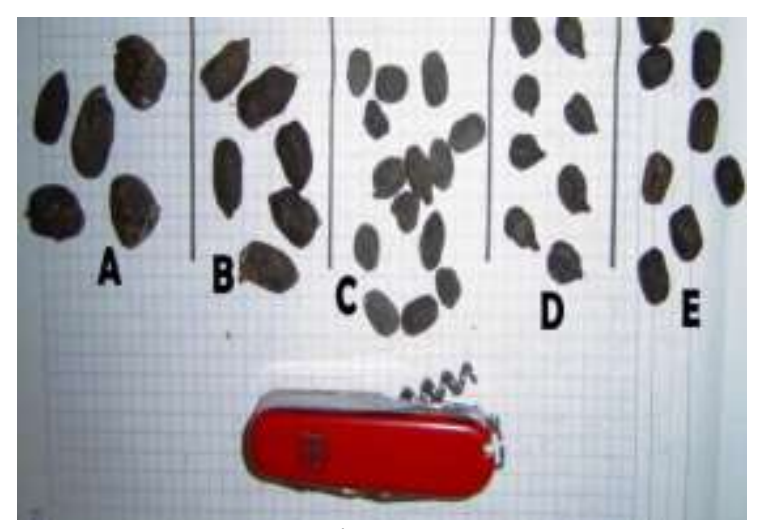

Figura 3. Morfología de las excretas de las diferentes especies de cérvidos.

A.- C. elaphus; B.- D. dama; C.- $O$. virginianus; D.- A. axis; E.- C. nippon 
Para la estimación de las poblaciones se tomaron en cuenta los promedios de tasas de defecación de las diferentes especies de cérvidos (Tabla 1), de acuerdo a una revisión bibliográfica realizada (Eberhardt y Van Etten, 1956; Neff et al.; 1965., Bailey y Putman, 1981; Dinestein, et al., 1982; Rollins et al., 1984; Massei y Genov, 1998; Mayle et al., 2000; Marques et al., 2001; Pérez-Mejía et al., 2004).

Tabla 1. Tasas de defecación de las diferentes especies de cérvidos

\begin{tabular}{|c|c|c|}
\hline $\begin{array}{l}\text { Especie de } \\
\text { cérvido }\end{array}$ & $\begin{array}{c}\text { *Tasa } \\
\text { diaria de } \\
\text { defecación }\end{array}$ & $\begin{array}{c}\text { Fuente } \\
\text { bibliográfica }\end{array}$ \\
\hline $\begin{array}{l}\text { Venado cola } \\
\text { blanca } \\
\text { (Odocoileus } \\
\text { virginianus) }\end{array}$ & 17 & $\begin{array}{l}\text { PÉREZ-MEJÍA } \\
\text { et al., } 2004\end{array}$ \\
\hline $\begin{array}{ll}\text { Ciervo } & \text { rojo } \\
\text { (Cervus } & \\
\text { elaphus) } & \end{array}$ & 12.5 & $\begin{array}{lll}\text { NEFF } & \text { et } & \text { al. } \\
1965 & & \end{array}$ \\
\hline $\begin{array}{l}\text { Venado } \\
\text { (Axis axis) }\end{array}$ & 20.3 & $\begin{array}{|lr|}\text { DINESTEIN } e t \\
\text { al., } & 1982 ; \\
\text { ROLLINS } & \text { et } \\
\text { al., } 1984 & \end{array}$ \\
\hline $\begin{array}{l}\text { Venado gamo } \\
\text { (Dama dama) }\end{array}$ & 18.9 & $\begin{array}{|lr|}\text { BAILEY } & \text { y } \\
\text { PUTMAN, } & \\
1981 ; & \\
\text { ROLLINS } & \text { et } \\
\text { al., } & 1984 ; \\
\text { MASSEI } & \text { y } \\
\text { GENOV, 1998; } \\
\text { MAYLE } \text { et } & \text { al. } . \\
2000 & \\
\end{array}$ \\
\hline $\begin{array}{l}\text { Venado } \\
\text { (Cervus } \\
\text { nippon) }\end{array}$ & 15 & $\begin{array}{|lr|}\text { ROLLINS } & e t \\
a l ., & 1984 ; \\
\text { MARQUES } & \text { et } \\
a l ., 2001 & \\
\end{array}$ \\
\hline
\end{tabular}

* Grupos de excretas por individuo
El segundo método utilizado, fue el de transecto en línea. Esa metodología consistió en el recorrido de transectos, con una longitud conocida. Se contaron los individuos que se lograran observar, registrando la distancia perpendicular de éstos. El análisis se realizó mediante el programa de cómputo Distance ${ }^{\mathrm{R}}$ (Thomas et al., 2005). Donde se considera que la probabilidad de detectar a los individuos disminuye a medida que aumenta la distancia perpendicular a la línea del trayecto. La fórmula para estimar la densidad es:

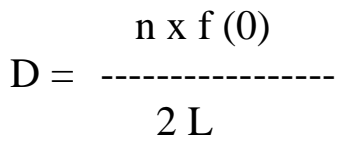

Donde $\mathrm{n}$ es el número de animales detectados; $\mathrm{f}(0)$ es la función probabilística de densidad a 0 metros de distancia perpendicular, y L es el largo total $(\mathrm{km})$ del transecto. Los supuestos a considerar en este método son (Thomas et al., 2005; Gallina et al; 2014):

Tener la certeza de que todo animal que esté próximo al centro del transecto será contado con una certidumbre del $100 \%$.

Los animales están fijos en el momento en que son detectados; no se mueven antes de ser detectados ni se cuentan más de una vez. Medir exactamente la distancia perpendicular del animal, al momento inicial de su detección.

La observación de cada individuo es un evento independiente.

Los recorridos de los transectos fueron nocturnos y diurnos, realizándose mediante caminatas y en vehículo (pick- 
up), con apoyo de binoculares. Los recorridos en vehículo fueron nocturnos, haciendo los avistamientos desde la parte más alta del automotor y con ayuda de lámparas (spot light) con una luminosidad de 500.000 candelas (Villarreal, 1999). Se recorrieron un total de $31,7 \mathrm{~km}$. Los recorridos diurnos iniciaban a las 7:00 am, y los recorridos nocturnos iniciaban 6:00 pm. Este muestreó se realizó simultáneamente en las fechas cuando se realizaba también el método indirecto a través de excretas.

Tabla 2. Resultados de densidades poblacionales mediante el método indirecto de Eberhardt y Van Etten.

\begin{tabular}{|c|c|c|c|}
\hline Especie & Ind//km² & $\begin{array}{c}\text { Desv. } \\
\text { estandar }\end{array}$ & $\begin{array}{c}\text { Total de } \\
\text { venados } \\
\text { en la } \\
\text { UMA }\end{array}$ \\
\hline $\begin{array}{c}\text { Cervus } \\
\text { elaphus }\end{array}$ & 3.9 & $.63+$ & 31 \\
\hline $\begin{array}{c}\text { Dama dama } \\
\text { Axis axis }\end{array}$ & 2.6 & $.49+$ & 21 \\
\hline $\begin{array}{c}\text { Cervus nippon } \\
\text { Odocoileus } \\
\text { virginianus }\end{array}$ & 2.6 & $.35+$ & 13 \\
\hline $\begin{array}{c}\text { Total de } \\
\text { individuos }\end{array}$ & & $.63+$ & 16 \\
\hline
\end{tabular}

\section{RESULTADOS}

En el método indirecto a través de excretas, los resultados muestran una densidad poblacional total de todas las especies de cérvidos de 99 individuos, donde el ciervo rojo fue la especie más abundante (Tabla 2). En el método directo a través del análisis del programa de computo Distance ${ }^{\mathrm{R}}$, los resultados muestran un total de 169 individuos de todas las especies, donde también el ciervo rojo fue el que presentó una densidad poblacional mayor (Tabla 3 ).

Tabla 3. Resultados de las densidades poblacionales de las diferentes especies de cérvidos obtenidas por el método directo de transecto lineal.

\begin{tabular}{|c|c|c|c|}
\hline Especie & Ind $/ \mathbf{k m}^{2}$ & $\begin{array}{c}\text { Porcentaje } \\
\text { de } \\
\text { coeficiente } \\
\text { de } \\
\text { variación } \\
\text { al } 95 \%\end{array}$ & $\begin{array}{c}\text { Total de } \\
\text { ejemplares } \\
\text { en la UMA }\end{array}$ \\
\hline $\begin{array}{l}\text { Cervus } \\
\text { elaphus }\end{array}$ & 6.1 & 40.8 & 49 \\
\hline Dama dama & 4.7 & 47 & 38 \\
\hline Axis axis & 3.8 & 49 & 30 \\
\hline Cervus nippon & 3.8 & 54.6 & 30 \\
\hline Odocoileus & 2.8 & 44.5 & 22 \\
\hline virginianus & & & \\
\hline Total & & & 169 \\
\hline
\end{tabular}

\section{DISCUSIÓN Y CONCLUSIÓN}

De acuerdo a los resultados obtenidos en los dos tipos de muestreos que se realizaron, podemos concluir diferencias en las estimaciones de la densidad poblacional de las cinco especies de ciervos; sin embargo ambos métodos no pueden ser comparados estadísticamente, por ser diferentes.

Sin embargo se consideró que el método directo de transecto en línea mediante observación, no es tan confiable en cuanto a sus resultados, pues las

Revista Científica Biológico Agropecuaria Tuxpan 5 (1) 
observaciones de individuos fueron pocas, los coeficientes de variación son altos y los intervalos de confianza amplios. Por consiguiente se eligieron como resultados más confiables, los obtenidos a través del método indirecto por excretas; ya que los hallazgos y las identificaciones de heces por especie de cérvido fueron exitosas, a través de su morfología (Rollins et al. 1984) y con apoyo de los guías locales, quienes cuentan con gran experiencia; además de mostrar resultados estadísticos más confiables. Además, en los transectos recorridos hubo siempre un número elevado de muestras identificadas; y las densidades estimadas fueron menores, en comparación con el método directo, lo cual permite no sobrestimar la población. La época del año en que se realizó este muestreo era la ideal, durante los meses de agosto, septiembre y octubre (Villarreal, 1999), ya que están presentes los cervatos que nacieron ese año, y no es temporada de gestación de las hembras, lo que permite realizar una mejor estimación.

Se considera que la capacidad de carga $(K)$ del hábitat para la densidad de la población estimada es insuficiente, considerando que el coeficiente de agostadero es de una unidad animal (UA)/12 ha (Villarreal, 1999), para el caso del venado cola blanca. De acuerdo a nuestros resultados hay 2.5 individuos/12 has. en la UMA, siendo la gran mayoría individuos de especies exóticas, que tienen mayor peso y tamaño, y por consiguiente requieren mayor espacio y alimento que el venado cola blanca; por lo tanto la competencia entre individuos de distintas especies es alta. Los cérvidos exóticos pueden estar generando efectos negativos sobre los árboles, y en la composición y regeneración del bosque. El venado cola blanca es una especie muy especializada y selectiva con una baja proporción de rumen: peso corporal, por lo que requiere dietas de alta calidad compuesta de arbustos y hierbas. En consecuencia, son incapaces de cambiar su dieta a gramíneas, propiciando el desplazamiento de esta por los exóticos. Así mismo la presencia de los cérvidos exóticos representa una amenaza para la población del venado nativo y de otras especies de mamíferos, por ser posibles portadores y transmisores de enfermedades y parásitos. C. nippon y A. axis son especies que presentan agresividad y territorialismo; factores que le permiten desplazar a especies nativas y establecerse en los sitios en que se dispersan (Álvarez et al., 2008). Un ejemplo de consecuencias negativas por sobrepoblación de herbívoros, es en el parque Yellowstone en Estados Unidos, donde se ha generado estragos en el hábitat, modificando del paisaje, generando cambios y evitando la sucesión natural de los bosques (Douglas et al, 2003).

Por lo anteriormente expuesto, sugerimos excluir a los cérvidos exóticos de esa

UMA, como una medida de conservación; protegiendo a ese ecosistema, así como a las especies nativas como el venado cola blanca y el teporingo entre otros animales, de acuerdo a la legislación manifiesta en la Ley General de Vida Silvestre de México (SEMARNAP, 2000), Capítulo V, Artículo 27 que dice: "El manejo de ejemplares y poblaciones exóticos solo se podrá llevar a cabo en condiciones de confinamiento, de acuerdo con un plan de manejo que deberá ser previamente aprobado por la Secretaría, y en el que se establecerán las condiciones de seguridad y de contingencia, para evitar los efectos negativos que los ejemplares y poblaciones exóticos pudieran tener para 
Romero-Castañón et al., 2017

la conservación de los ejemplares y poblaciones de especies nativos de la vida silvestre y su hábitat". Esto toma singular importancia, ya que la UMA colinda con una ANP, que además es símbolo de la tradición y la cultura mexicana, ya que en la Sierra Nevada se ubican dos de las montañas con mayor altitud en México, los denominados volcanes sagrados la Iztaccíhuatl y el Popocatépetl (Glokner, 1996; Villarreal, 1998).

\section{LITERATURA CITADA}

Alvarez-Romero,J.G.; Medellín, R.A.; Oliveras de Ita, A.; Gómez de Silva, H.; Sánchez, O. 2008. Animales exóticos en México: una amenaza para la biodiversidad. Editorial Comisión Nacional para el Conocimiento y Uso de la Biodiversidad, Instituto de Ecología, UNAM, Secretaría de Medio Ambiente y Recursos Naturales, México, D.F. 518 pp.

Bailey,R. E.; Putman, R.J. 1981. Estimation of fallow deer (Dama dama) populations from faecal accumulation. Journal of applied ecology. 18: 697-702 https://doi.org/10.2307/2402361

Burnham, K. P.; Anderson, D.R.; Laake, J.L. 1980. Estimation of density from line transect sampling of biological populations. Wildlife Monographs. 72: 1-202 https://doi.org/10.2307/3800636 Clutton-Brock, J. 2002. Mamíferos. Manual de identificación. Ediciones Omega. Barcelona. 400 pp.

Dinestein, E.; Dublin. H. 1982. Daily defecation rate of captive axis deer. 1982. Journal of Wildlife Management. 46 (3): 1 833-835. https://doi.org/10.2307/3808586

Douglas, W.; Rolf, O.; Peterson, B.; Douglas, B. 2003. Yellowstone after wolves. BioScience. 53 (4):

330-340

https://doi.org/10.1641/0006-3568

Eberhardt, L. L.; Van Etten, R.C. 1956.

Evaluation of the pellet group count as a deer census method. Journal of Wildlife Managament. 20: $70-74$

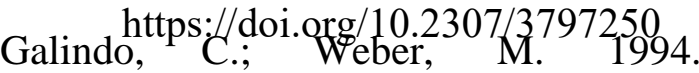
Translocation of deer subepecies: Reproductive Implications. Wildlife Society Bulletin. 117-120 pp.

Gallina, S.; Mandujano, S.; Villarreal, E. 2014. Monitoreo y manejo del venado cola blanca: Conceptos y métodos. Editorial Instituto de Ecología, A.C. y Benemérita Universidad Autónoma de Puebla. Xalapa, Ver. México. 218 pp.

Harrington, R. 1985. Evolution of the Cervidae. Págs. 3-11 en: Drew, K.; Fenessy (eds). Biology of deer production. The Royal Society. of New Zealand.

Glokner, J. 1996. Los volcanes sagrados, mitos y realidades en Popocatépetl y la Iztaccíhuatl, México: Ed. Grijalbo, 201 pp.

INEGI (Instituto Nacional de Estadística, Geografía e Informática). 2000. Síntesis Geográfica del Estado de Puebla; Libro Electrónico.

Marques, F. F.; Buckland, S. T.; Goffin, D.; Dixon, C., Borchers, D.; Mayle, B.; Peace, A. 2001. Estimating deer abundance from line transect surveys of dung: sika deer in Southern Scotland. Journal of Applied Ecology. 38: 349-363. https://doi.org/10.1046/j.1365-26

Massei, G.; Genov, P. V. 1998. Fallow deer (Dama dama) winter defecation rate in a mediterranean area. Journal of Zoology London. 245: 209-210

https://doi.org/10.1111/j.1469-799

Mayle, B. A.; Putman, R. J.; Wyllie, I. 2000. The use of trackway counts to establish an index of deer 
presence. Mammal Review. 3 y 4: 233237 https://doi.org/10.1046/j.1365-290

Neff, D. J.; Wallmo, O. C.; Morrison, D. C. 1965. A determination of defecation rate for elk. Journal of Wildlife Management. 29: 406407 https://doi.org/10.2307/3798454

Pérez-Mejía, S.; Mandujano, S.; Martínez-Romero, L. E. 2004. Tasa de defecación del venado cola blanca, Odocoileus virginianus mexicanus, en cautividad en Puebla, México. Acta Zoológica Mexicana. 20 (3): 167-170

Putman, R. (ed) 1988. The Natural History of Deer. Cornell University Press. Ithaca, New York.

Ramírez, R. G. 2004. Nutrición del Venado Cola Blanca. Universidad Autónoma de Nuevo León; Unión Ganadera Regional de Nuevo León; Fundación PRODUCE Nuevo León, A. C. Eds.: 240 pp.

Rollins, D.; Bryant, F.; Montandon, R. 1984. Fecal ph and defecation rates of eight ruminants fed known diets. Journal of Wildlife Management 48 (3): 807-813 https://doi.org/10.2307/3801427

Sanders, C. L. 1963. Habitat preferences of the white.tailed deer and several exotic ungulates in south Texas. Ecology. 44: 803-806 https://doi.org/10.2307/1933036

SEMARNAT, 2000. Ley General de Vida Silvestre. Editorial Secretaría de Medio Ambiente y Recursos Naturales. México

Thomas, L.; Laake, J.L.; Strindberg, S., Marques, F.F.C.; Buckland, S.T.; Borchers, D.L.; Anderson, D.R., Burnham, K.P., Hedley, S.L., Pollard, J.H., Bishop, J.R.B. and Marques, T.A. 2005. Distance 5.0. Release " $x$ "1. Research Unit for Wildlife Population
Assessment, University of St. Andrews, UK.

Van Soest, P. J. 1994. Nutritional Ecology of the Ruminant. Second edition. Cornell University Press. $476 \mathrm{pp}$.

https://doi.org/10.7591/9781501732

Villarreal, O. A. 1998. Nueva Alerta Don Gregorio: Los Cérvidos Exóticos en el Popo-Izta. En: Memorias del "VI Simposio sobre Venados de México" UNAM. ANGADI. Instituto de Ecología, A.C. 83-88

Villarreal. J. G.. 1999. Venado Cola Blanca. Manejo y Aprovechamiento Cinegético. Editorial Unión Ganadera Regional de Nuevo León. Monterrey, N. L. México. Impresora Monterrey, S. A. de C. $\mathrm{V}$.

Weber,M.; Galindo-Leal, C. 1992. Distocia en venado cola blanca: Informe de un caso reincidente. Veterinaria Mexico. 23(1)69-71

Weber, M. 1993. Ganaderìa de ciervos: ¿Alternativa de producción animal o amenaza a la conservación de la fauna nativa? Agrociencia. 3 (2) 99-113..

Weber,M.; García-Marmolejo, G.; ReynaHurtado, R. 2006. The tragedy of the commons: Wildlife Management Units in the Southeaestern Mexico. Wildlife Society Bull. 34 (5): 1480-1488 https://doi.org/10.2193/0091-7648 


\section{(c) (1)}

Este tex to está protegido por una licencia licencia Creative Commons 4.0

Usted es libre para Compartir —copiar y redistribuir el material en cualquier medio o form ato-y Adaptar el documento —remezclar, transformar y crear a partir del material- para cualquier propósito,, incluso para fines comerciales, siempre que cumpla la condición de:

Atribución: Usted debe dar crédito a la obra original de manera adecuada, proporcionar un enlace a la licencia, e indicar si se han realizado cam bios. Puede hacerlo en cualquier form a razonable, pero no de form a tal que sugiera que tiene el apoyo del licenciante o 10 recibe por el uso que hace de la obra.

Resumendelicencia - Textocompletodelalicencia 